\title{
The Effect of Preoperative AchR-Ab Level to the Prognosis in Operated Myasthenia Gravis Patients
}

\author{
Tevfik Ilker Akcam ${ }^{1 *}$, Ali Ozdil1, Onder Kavurmaci1', Ayse Gul Ergonul1', Kutsal Turhan1, \\ Alpaslan Cakan1, Ufuk Cagirici1, Ayse Nur Yuceyar²
}

\author{
${ }^{1}$ Department of Thoracic Surgery, School of Medicine, Ege University, Izmir, Turkey \\ ${ }^{2}$ Department of Neurology, School of Medicine, Ege University, Izmir, Turkey \\ Email:*tevfikilkerakcam@hotmail.com
}

How to cite this paper: Akcam, T.I., Ozdil, A., Kavurmaci, O., Ergonul, A.G., Turhan, K., Cakan, A., Cagirici, U. and Yuceyar, A.N. (2017) The Effect of Preoperative AchR-Ab Level to the Prognosis in Operated Myasthenia Gravis Patients. Open Journal of Thoracic Surgery, 7, 62-69. https://doi.org/10.4236/ojts.2017.74009

Received: November 13, 2017 Accepted: December 16, 2017 Published: December 19, 2017

Copyright $\odot 2017$ by authors and Scientific Research Publishing Inc. This work is licensed under the Creative Commons Attribution International License (CC BY 4.0).

http://creativecommons.org/licenses/by/4.0/

\begin{abstract}
Objective: Seropositive myasthenia gravis (MG) depends on the presence of acetylcholine receptor antibodies (AchR-Ab) against nicotinic acetylcholine receptors at the postsynaptic neuromuscular junction. In this study, we investigated the effect of AchR-Ab levels to symptoms and treatment in the MG patients underwent surgery for thymic pathology. Materilas and Methods: The records including level of preoperative AchR-Ab, type of thymic pathology, the changes of symptoms and treatment after surgery for thymic pathology of 37 MG patients between January 2007 and December 2015 have been viewed retrospectively. Results: The mean age of 37 patients (21 females, 16 males) was $40.2 \pm 14.9$ years (range, 18 to 75 years). The mean of the level of AchR-Ab was $144.7 \pm 427.6 \mathrm{nmol} / \mathrm{L}$ (range, 0.1 to $1806 \mathrm{nmol} / \mathrm{L}$ ). In the patient group including the reduced use of anticholinesterase after surgery the mean of level of AchR-Ab was $241.3 \mathrm{nmol} / \mathrm{L}$ while it was 10.8 $\mathrm{nmol} / \mathrm{L}$ in the patient group including the non-reduced use of anticholinesterase $(p=0.082)$ after a mean follow-up period of 23 months postoperatively. The mean levels were $246.7 \mathrm{nmol} / \mathrm{L}$ and $8.5 \mathrm{nmol} / \mathrm{L}$ for the reduced and non-reduced use of corticosteroid patient groups, respectively $(\mathrm{p}=$ 0.001 ). In the 25 patients with fewer symptom after surgery the mean of the AchR-Ab level was $205.3 \mathrm{nmol} / \mathrm{L}$ while it was $18.3 \mathrm{nmol} / \mathrm{L}$ in the patients without any changes $(p=0.071)$. Conclusion: We concluded that the preoperative level of AchR-Ab was associated with postoperative dosage of anticholinesterase and corticosteroids and severity of symptoms postoperatively. We think that the levels of AchR-Ab titers can be a marker for the efficacy of thymic surgery.
\end{abstract}




\section{Keywords}

AchR-Ab, Myasthenia Gravis, Thymectomy

\section{Background}

Myasthenia gravis (MG) is an autoimmune and neuromuscular disease characterized by fluctuating muscle weakness which increases with the repetitive activity of the voluntary muscles and disappears or decreases with rest and the use of anticholinesterase agents. The prevalence of MG is 25 - 100/1.000.000 [1] [2] [3]. It is most commonly seen in 2.-3. and 5. decades in females and 5. decades in males. Mostly, it depends on the presence of acetylcholine receptor antibodies (AchR-Ab) against nicotinic acetylcholine receptors at the postsynaptic neuromuscular junction. These antibodies are highly specific for MG. AchR-Ab is positive in $85 \%$ of generalized MG patients and nearly all of patients with thymoma but it is positive only in 50\% of ocular MG patients [1] [3] [4] [5]. Thymic pathology exists in $10 \%-20 \%$ of MG patients and AchR-Ab positivity shows variability in these pathologies except thymoma. However, the prevalence of MG is about $20 \%-50 \%$ in patients with thymoma [2] [3] [6]. Any study investigating the relation between AchR-Ab level and effectivity of surgery was not reported in the literature. In this study we aimed to evaluate the effect of AchR-Ab levels to the postoperative symptoms and treatment in $M G$ patients undergoing surgery for thymic pathology.

\section{Material and Methods}

The records of 37 patients underwent extended mediastinal resection for thymic pathology between January 2007 and December 2015 were evaluated retrospectively. All of the patients had MG diagnosis by physical examination, electromyography (EMG) and AchR-Ab level which were performed by Neurology department. Non-MG patients were excluded from the study. The treatment and follow-up of patients were designed by Neurology department and anticholinesterase and corticosteroid treatment were started to all of the patients. The patients with thymic pathology which had been detected on radiological examinations were referred to our clinic for surgery. Extended thymectomy or thymothymectomy extending from the lower end of the thyroid to the diaphragm and laterally from the bilateral mediastinal pleura to the phrenic nerves was performed to all of the patients. Patients were continued to follow in appropriate intervals (mean of follow-up was 23 months) in terms of preoperative and postoperative severity of symptoms and dosage of anticholinesterase and corticosteroids. Beside these sex, date of birth, comorbid disease, main symptom, AchR-Ab levels, the duration of symptoms from the onset to the surgery, pathological results were analyzed. Informed consent was obtained from all individual participants included in the study. The study protocol has been approved by the 
Institutional Review Board of Ege University Medical School, registered to No. 16-6/44 and has been performed in accordance with the ethics standards laid down in the 1964 Declaration of Helsinki and its later amendments.

\section{Statistical Analyses}

Data analysis was performed using SPSS version 18.0 for Windows (SPSS, Chicago, IL, USA). Non-parametric tests (Mann-Whitney U test) were performed to determine the relationship between AchR-Ab levels and preoperative and postoperative severity of symptoms and the dosage of preoperative and postoperative Anticholinesterase and corticosteroids. " $p$ " values of less than 0.05 were considered to be statistically significant.

\section{Results}

The mean age of 37 patients including 21 (56.8\%) females and 16 (43.2\%) males was $40.2 \pm 14.9$ years (range, 18 to 75 years). The patients were under follow-up with the diagnosis of MG with a mean time of $8.3 \pm 10.1$ months (range, 1 to 48 months). The pathologies were reported as thymoma in 25 (67.6\%) patients, thymic hyperplasia in 12 (32.4\%) patients who all underwent extended resection for thymic pathology. The mean of AchR-Ab titers of all patients was $144.7 \pm$ $427.6 \mathrm{nmol} / \mathrm{L}$ (range, 0.1 to $1806 \mathrm{nmol} / \mathrm{L}$ ). The patients with the AchR-Ab titers less than $0.4 \mathrm{nmol} / \mathrm{L}$ were accepted "seronegative MG" while the patients with the AchR-Ab titers more than $0.4 \mathrm{nmol} / \mathrm{L}$ were accepted as "seropositive MG". The thymic pathology was thymic hyperplasia in all of the seronegative MG patients. There were ocular beginning in nineteen patients and generalized beginning in 18 patients according to the evaluation of initial symptoms. Median sternotomy were performed to 33 patients while VATS thymectomy/thymothymectomy were performed to 4 patients as the widened use of VATS. Mortality was seen in two patients in the early postoperative period and these patients were excluded from the study (Table 1).

Patients were continued to follow in appropriate intervals (mean of follow-up was 23 months). In follow period it was determined that the need of anticholinesterase had been decreased in 21 patients, no change in 12 patients and the need of increase in dosage in 2 patients. Similar distribution was determined for the dosage of corticosteroids. Twenty-five patients expressed that their symptoms had been regressed after surgery while 10 expressed no changes. The mean of AchR-Ab titers of patients with the decreased dosage of anticholinesterase was $241.3 \mathrm{nmol} / \mathrm{L}$ whereas it was $10.8 \mathrm{nmol} / \mathrm{L}$ of the patients with no change and the difference was not significant $(\mathrm{p}=0.082)$. The means of AchR-Ab titers of the patients with decreased dosage of corticosteroids and with no change were 246.7 and $8.5 \mathrm{nmol} / \mathrm{L}$, respectively. The difference was statistically significant with $\mathrm{p}$ value of 0.001 (Figure 1). The difference was not significant but it was very close to significance when the means of AchR-Ab titers of the patients with the decreased severity of symptoms and with no changes in symptoms were compared (the means were $205.3 \mathrm{nmol} / \mathrm{L}$ and $18.3 \mathrm{nmol} / \mathrm{L}$, respectively and $\mathrm{p}=0.071$ ) 
Table 1. Patients' demographic data.

\begin{tabular}{ccccc}
\hline & & “n” & “\%” & $\begin{array}{c}\text { Mean AchR-ab } \\
\text { level (mmol/L) }\end{array}$ \\
\hline Age & Se, 24/years & 37 & & \\
& Male & 16 & $43.2 \%$ & 133.64 \\
Symptom & Female & 21 & $56.8 \%$ & 153.26 \\
& Ocular & 19 & $51.4 \%$ & 106.38 \\
Histology & Generalized & 18 & $48.6 \%$ & 185.31 \\
& Thymic hyperplasia & 12 & $32.4 \%$ & 136.28 \\
Operation type & Thymoma & 25 & $67.6 \%$ & 148.86 \\
& Sternotomy & 33 & $89.2 \%$ & 162.02 \\
Concomitant & VATS & 4 & $10.8 \%$ & 2.57 \\
Diseases & Positive & 8 & $21.6 \%$ & 19.9 \\
& Negative & 29 & $78.4 \%$ & 179.23 \\
Receptor & Positive & 33 & $89.2 \%$ & 162.29 \\
& Negative & 4 & $10.8 \%$ & $<0.1$ \\
\hline
\end{tabular}

n; patient number, VATS: video assisted thoracoscopic surgery.

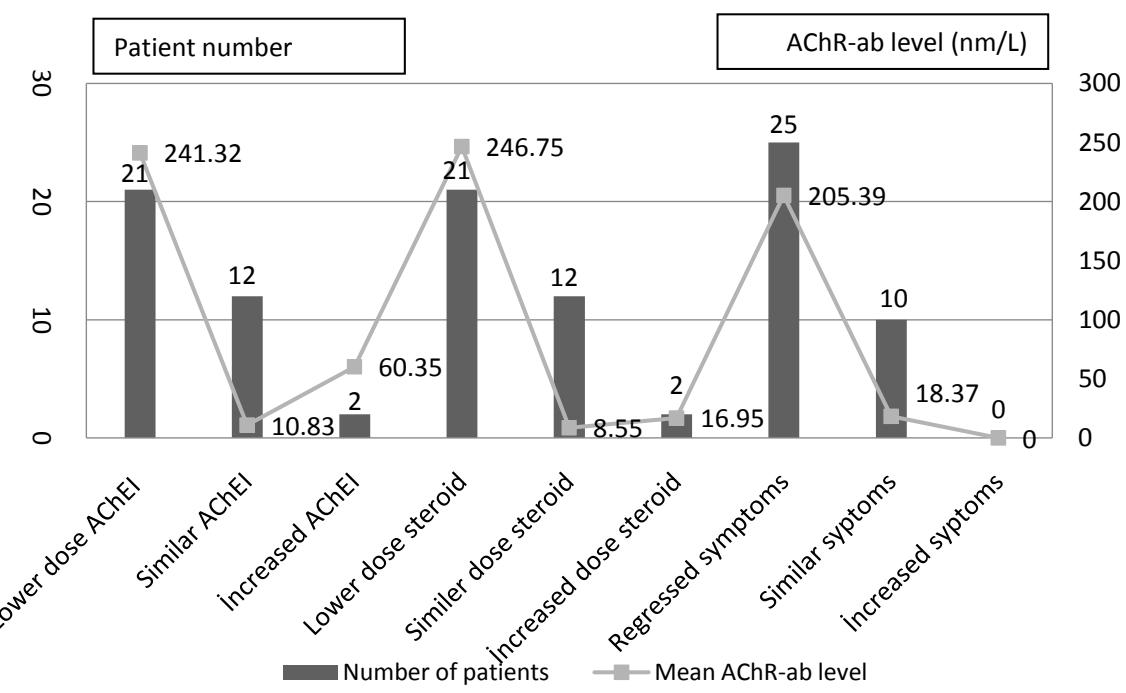

Figure 1. Treatment response-antibody level-number of patients. AChEI; Acetylcholinesterase inhibitor, AChR-ab; Acetylcholine receptor antibody.

(Table 2). The mean of the AchR-Ab titers was $160.1 \mathrm{nmol} / \mathrm{L}$ in the patients with the pathology of thymoma and it was $136.82 \mathrm{nmol} / \mathrm{L}$ in the patients with thymic hyperplasia $(\mathrm{p}=0.768)$. The AchR-Ab titers of patients with ocular MG (mean: $106.3 \mathrm{nmol} / \mathrm{L}$ ) was significantly $(\mathrm{p}=0.03)$ lesser than the AchR-Ab titers of the patients with generalized MG (mean: $206.1 \mathrm{nmol} / \mathrm{L}$ ).

\section{Conclusions}

The treatment of MG can be hold in two parts as medical and surgical. The 
Table 2. Preoperative acetylcholine receptor antibodies (AchR-Ab) levels in postoperative patients' condition.

\begin{tabular}{cccccc}
\hline & & Regressed & Similar & Increased & “p” \\
\hline \multirow{2}{*}{ Anticholinesterase use } & Patients/n & $21(56.8 \%)$ & $12(32.4 \%)$ & $2(5.4 \%)$ \\
& Mean AchR-Ab/nmol/L & $241.32 \pm 553.25$ & $10.83 \pm 8.24$ & $60.35 \pm 61.73$ & 0.082 \\
Corticosteroid use & Patients/n & $21(56.8 \%)$ & $12(32.4 \%)$ & $2(5.4 \%)$ \\
Severity of symptoms & MeanR-Ab/nmol/L & $246.75 \pm 551.18$ & $8.55 \pm 12.48$ & $16.95 \pm 0.35$ & 0.001 \\
$\begin{array}{c}\text { after surgery } \\
\text { Patients/n }\end{array}$ & $25(67.6 \%)$ & $10(27 \%)$ & - & - \\
\end{tabular}

medical treatment mainly includes anticholinesterase, immunosupressive agents (corticosteroids, azathiopurine, etc.), plasmapheresis and intravenous immunoglobulin (IVIG) but single drug treatment almost has never been successful and used for the treatment. Because of this reason the treatment of MG is a multimodal regimen [1] [3] [7]. In 1939 Blalock [8] mentioned that mediastinal exploration and resection should be performed to $M G$ patients whether thymoma had been detected or not. Different results were reported for the MG patients undergoing mediastinal tumor resection since this time [9]. MG is the most common neurologic disease accompanying to thymomas [3] [6]. The remission rates after resection are higher especially in MG patients with thymoma [1] [10]. Therefore there is no suspicion about the necessity of surgery for MG patients with thymoma. In the presence of thymoma there is an indication for thymothymectomy (recommendation grade A) whereas thymectomy is recommended for the increase in the probability of remission and regression [11]. Thymectomy is recommended in retrospective studies for the seronegative, generalized, thymic hyperplasia patients between puberty and age of 60 years considering that they generally benefit from surgery [12]. However as seen in different studies, there is no consensus about surgical resection of the mediastinal lesion except thymoma [10] [13]. Because of this reason detailed information about the effectiveness of surgery, interaction of the surgery with other treatment methods and the changes of the medical treatment after surgery must be given to MG patients without thymoma. In a prospective study including $75 \mathrm{MG}$ patients without thymoma, Richard K et al. [14] reported that complete remission was seen in 21 patients who underwent robotic surgery and no progression was seen in the other patients. A review promoting this study and published recently mentioned that thymic pathology did not influence postoperative remission and should not influence the indication for thymectomy and added that a prospective study about this subject will be ended soon [9].

Ocular complaints are the initial symptoms in close to the half of the MG patients while generalized complaints are the initial symptoms in the other half. In a little while bulbar and extremity muscles symptoms are added to ocular findings and this situation is called as generalized MG. Generalized MG findings are observed in $40 \%$ - 50\% of ocular MG in the following first one or two years [7] 
[15]. The patients without any generalized finding added in two years are accepted as pure ocular MG. Thymectomy is preferred as first or second step of treatment in the patients with pure ocular MG. There is no consensus about the effectiveness of the thymectomy for the treatment of ocular MG patients in the literature [1] [15] [16]. While some researchers mention that these patients benefit symptomatically from surgery, the others mention no difference between medical treatment and surgery [15] [16]. In our study, similar distribution was seen in terms of ocular symptoms and $51.3 \%$ of patients had ocular complaints. Generalized findings accompanied to all of patients with ocular beginning. Pure ocular MG patients were not included to the study.

As mentioned earlier AchR-Ab is a very specific marker for MG. But in some patients it cannot be detected or can be measured in very low titers in blood. These patients are called as seronegative MG. Pure ocular MG cases compose a large part of the seronegative MG [5]. Also in our series 4 patients with seronegativity had ocular symptoms. Muscle-specific receptor tyrosine kinase (MuSK-Ab) can be positive in 30\% - 40\% of seronegative MG patients but MuSK-Ab levels could not be invastigated in these 4 cases. The pathogenesis of Anti-MuSK could not be defined clearly but it is thought to be that it can reduce the number of AchR-Ab functionally by preventing the clustering of the AchR-Ab at the end plate [7] [17]. Thymectomy is controversial for Anti-MuSK seropositive patients because of no benefit of these patients from surgery.

Thymic surgery changes in time by the use VATS in recent years. Although studies about the treatment approaches, prognosis and clinical classification were reported the surgical approach varies by the developing surgical techniques and technological devices [13] [18]. Especially with the use of VATS for mediastinal surgery in 1990s different processes including video thoracoscopic simple mediastinal cyst resection to robotic thymoma resections had been performed [14] [19] [20]. Better exposure and lighting, shorter hospital stay, better cosmetic look and less complication rates are shown as the advantages of minimal invasive surgery [14] [20]. Most of the members of The Thymic Working Group of the European Association for Cardio-Thoracic Surgery (EACTS) attending to a questionnaire mentioned that they had been preferring trans-pleural approach for the thymomas under $4 \mathrm{~cm}$ [21]. Also in a multi-centered study it was reported that robotic thymic surgery had been feasible and safe for early stage thymomas but also it was mentioned that the long-term results must be followed [19]. In our study most of the patients underwent trans-sternal surgery while VATS was performed to four patients with a thymoma smaller than $4 \mathrm{~cm}$ especially in the recent years and no complication was seen in these cases.

The number of studies investigating AchR-Ab titer level and its prognostic significance is limited in the literature. It is known that the severity of disease is not associated with the level of antibody and the follow-up of levels of antibody is not a maker for the evaluation of response to treatment. We did not meet any study especially about the effect of levels of AchR-Ab titers on the patients un- 
derwent mediastinal surgery for MG. In our study we observed that preoperative AchR-Ab of MG patients was directly related with the postoperative severity of symptoms and the dosage of anticholinesterase and corticosteroids. We convinced that high preoperative AchR-Ab titers can be a good prognostic marker for utilization from surgery in short period. It must be keep in mind that especially in patients with low AchR-Ab titers there cannot be a change in the severity of symptoms and the dosage of medical treatment postoperatively.

\section{Conflict of Interest}

The authors declare that they have no conflict of interest.

\section{Ethical Board Approval}

The study protocol has been approved by the Institutional Review Board of Ege University Medical School, registered to No. 16-6/44 and has been performed in accordance with the ethics standards laid down in the 1964 Declaration of Helsinki and its later amendments.

\section{Informed Consent}

Informed consent was obtained from all individual participants included in the study.

\section{References}

[1] Silvesti, N.J. and Wolfe, G.I. (2012) Myasthenia Gravis. Seminars in Neurology, 32, 215-226. https://doi.org/10.1055/s-0032-1329200

[2] Cevik, A.G., Turhan, K., Cakan, A., Ozdil, A. and Cagirici, U. (2011) Myasthenia Gravis with Thymoma: Histopathologic Examination and Rate of Complication after Surgery. Journal of Clinical and Analytical Medicine, 2, 22-23. https://doi.org/10.4328/JCAM.205

[3] Vincent, A., Palace, J. and Hilton-Jones, D. (2001) Myasthenia Gravis. Lancet, 357, 2122-2128. https://doi.org/10.1016/S0140-6736(00)05186-2

[4] Aurangzeb, S., Tariq, M., Irshad, M., Badshah, M. and Khan, R.S. (2009) Relationship between Anti-Acetylcholine Receptor Antibody Titres and Severity of Myasthenia Gravis. Journal of the Pakistan Medical Association, 59, 289-292.

[5] Meriggioli, M.N. (2009) Myasthenia Gravis with Anti-Acetylcholine Receptor Antibodies. Frontiers of Neurology and Neuroscience, 26, 94-108. https://doi.org/10.1159/000212371

[6] Marx, A., Müller-Hermelink, H.K. and Ströbel, P. (2003) The Role of Thymomas in the Development of Myasthenia Gravis. Annals of the New York Academy of Sciences, 998, 223-236. https://doi.org/10.1196/annals.1254.025

[7] Conti-Fine, B.M., Milani, M. and Kaminski, H.J. (2006) Myasthenia Gravis: Past, Present, and Future. Journal of Clinical Investigation, 116, 2843-2854.

https://doi.org/10.1172/JCI29894

[8] Blalock, A., Mason, M.F., Morgan, H.J. and Riven, S.S. (1939) Myasthenia Gravis and Tumors of the Thymic Region: Report of a Case in Which the Tumor Was Removed. Annals of Surgery, 11, 544-561. 
https://doi.org/10.1097/00000658-193910000-00005

[9] Mao, Z., Hu, X., Lu, Z. and Hackett, M.L. (2015) Prognostic Factors of Remission in Myasthenia Gravis after Thymectomy. European Journal of Cardio-Thoracic Surgery, 48, 18-24. https://doi.org/10.1093/ejcts/ezu309

[10] Gronseth, G.S. and Barohn, R.J. (2000) Thymectomy for Non-Thymomatous Autoimmune Myasthenia Gravis. Neurology, 55, 7-15. https://doi.org/10.1212/WNL.55.1.7

[11] Skeie, G.O., Apostolski, S., Evoli, A., Gilhus, N.E., Illa, I., Harms, L., et al. (2010) European Federation of Neurological Societies. Guidelines for Treatment of Autoimmune Neuromuscular Transmission Disorders. European Journal of Neurolo$g y, 17,893-902$. https://doi.org/10.1111/j.1468-1331.2010.03019.x

[12] Meriggioli, M.N. and Sanders, D.B. (2009) Autoimmune Myasthenia Gravis: Emerging Clinical and Biological Heterogeneity. The Lancet Neurology, 8, 475-490. https://doi.org/10.1016/S1474-4422(09)70063-8

[13] Jaretzki, A., Barohn, R.J., Ernstoff, R.M., Kaminski, H.J., Keesey, J.C., Penn, A.S., et al. (2000) Myasthenia Gravis: Recommendations for Clinical Research Standards. Task Force of the Medical Scientific Advisory Board of the Myasthenia Gravis Foundation of America. The Annals of Thoracic Surgery, 70, 327-334.

https://doi.org/10.1016/S1474-4422(09)70063-8

[14] Freeman, R.K., Ascioti, A.J., Van Woerkom, J.M., Vyverberg, A. and Robison, R.J. (2011) Long-Term Follow-Up after Robotic Thymectomy for Nonthymomatous Myasthenia Gravis. The Annals of Thoracic Surgery, 92, 1018-1022.

https://doi.org/10.1016/j.athoracsur.2011.04.054

[15] Liu, Z., Feng, H., Yeung, S.C., Zheng, Z., Liu, W., Ma, J., et al. (2011) Extended Transsternal Thymectomy for the Treatment of Ocular Myasthenia Gravis. The Annals of Thoracic Surgery, 92, 1993-1999. https://doi.org/10.1016/j.athoracsur.2011.08.001

[16] Haines, S.R. and Thurtell, M.J. (2012) Treatment of Ocular Myasthenia Gravis. Current Treatment Options in Neurology, 14, 103-112. https://doi.org/10.1007/s11940-011-0151-8

[17] Vincent, A. and Leite, M.I. (2005) Neuromuscular Junction Autoimmune Disease: Muscle Specific Kinase Antibodies and Treatments for Myasthenia Gravis. Current Opinion in Neurology, 18, 519-525. https://doi.org/10.1097/01.wco.0000180660.57801.3f

[18] Masaoka, A., Monden, Y., Nakahara, K. and Tanioka, T. (1981) Follow-Up Study of Thymomas with Special Reference to Their Clinical Stages. Cancer, 48, 2485-2492. https://doi.org/10.1002/1097-0142(19811201)48:11<2485::AID-CNCR2820481123> 3.0.CO;2-R

[19] Marulli, G., Maessen, J., Melfi, F., Schmid, T.A., Keijzers, M., Fanucchi, O., et al. (2016) Multi-Institutional European Experience of Robotic Thymectomy for Thymoma. Annals of Cardiothoracic Surgery, 5, 18-25.

[20] Graza, A. and Woo, E. (2016) Video-Assisted Thoracoscopic Surgery versus Sternotomy in Thymectomy for Thymoma and Myasthenia Gravis. Annals of Cardiothoracic Surgery, 5, 33-37.

[21] Clucchi, M., Van Schil, P., Schmid, R., Rea, F., Melfi, F., Athanassiadi, K., Zielinski, M., Treasure, T. and EACTS Thymic Working Group (2012) Thymectomy for Thymoma and Myasthenia Gravis. A Survey of Current Surgical Practice in Thymic Disease amongst EACTS Members. Interactive CardioVascular and Thoracic Surgery, 14, 765-770. https://doi.org/10.1093/icvts/ivs046 